\title{
Lupus Eritematoso Sistêmico: Novos Paradigmas e Manejo no Atendimento Emergencial - Revisão de Literatura
}

\author{
Systemic Lupus Erythematosus: New Paradigms and Management \\ in the Emergency Care - Literature Review
}

\author{
Rita Mira ${ }^{1}$, Luan Guanais Soriano ${ }^{1}$, Catharine Bittencourt Cunha ${ }^{1 *}$, \\ Ramon Campos Nascimento ${ }^{1}$ \\ ${ }^{1}$ Serviço de Pediatria do Hospital Santa Izabel; Salvador, Bahia, Brasil
}

Correspondence addresses: Dra. Catharine B. Cunha catharine.bc@gmail.com

Received: December 14, 2019

Revised: January 13, 2020

Accepted: January 27, 2020

Published: March 31, 2020

Data Availability Statement: All relevant data are within the paper and its Supporting Information files.

Funding: This work was the result of author's initiative. There was no support of research or publication funds.

Competing interests: The authors have declared that no conflit of interests exists.

\section{Copyright}

(C) 2020 by Santa Casa de Misericórdia da Bahia. All rights reserved.

ISSN: 2526-5563
O lupus eritematoso sistêmico é uma doença autoimune heterogênea, resultante de desregulação complexa do sistema imunológico inato e adaptativo, afetando potencialmente qualquer órgão e / ou tecido. O lupus eritematoso sistêmico é uma doença imunemediada cuja patogênese originária resulta em exposição a autoantígeno, dando origem a numerosos anticorpos auto-reativos de especificidades antigênicas variadas que, juntamente com uma infinidade de citocinas, são consideradas efetoras da atividade da doença. Os regimes de tratamento existentes estão associados a vários efeitos colaterais debilitantes, com pacientes mais jovens em risco aumentado devido à duração do tratamento. Este trabalho teve como objetivo revisar e resumir sistematicamente a literatura disponível sobre a temática do lupus eritematoso sistêmico na população pediatrica através da revisão sistemática por meio de artigos disponibilizados atraves do PubMed e contextualizar a temática por meio de um relato de caso clínico.

Palavras-chave: Lúpus Eritematoso Sistêmico; Tratamentos; Pediatria.

Systemic lupus erythematosus is a heterogeneous autoimmune disease, resulting from complex dysregulation of the innate and adaptive immune systems, potentially affecting any organ and/or tissue.Systemic lupus erythematosus is an immunemediated disease whose originating pathogenesis results in autoantigen exposure giving rise to numerous autoreactive antibodies of varying antigenic specificities that along with a myriad of cytokines are thought to be effectors of disease activity.Existing treatment regimens are associated with numerous debilitating side effects, with younger patients at increased risk due to the duration of their disease. This study aimed reviewing and summarizing the available literature of juvenile-onset systemic lupus erythematosus by using articles on PubMed and a case report was related to elucidate the disease. Keywords: Systemic Lupus Erythematosus; Treatment; Pediatrics.

\section{Introdução}

O Lúpus Eritematoso Sistêmico (LES) é uma doença crônica multissistêmica de caráter autoimune com uma grande variação de etiopatogenia, manifestações clínico-laboratoriais e prognóstico. ${ }^{1}$ O LES apresenta etiologia ainda desconhecida e caracteriza-se pelo aumento da atividade do sistema imunológico com produção de autoanticorpos e 
deposição de imunocomplexos, que podem atingir e agredir diversos sistemas, de forma simultânea ou progressiva. ${ }^{2} \mathrm{~A}$ doença possui um amplo diagnóstico diferencial, especialmente em fases iniciais, o que dificulta a identificação precoce da doença. ${ }^{3}$

A prevalência é maior na população feminina (cerca de cinco a nove vezes mais comum do que em homens, a depender da população estudada), que pode ser justificada pela presença de variações hormonais. Entretanto, fatores ambientais, hereditários e emocionais têm sido relacionados com o surgimento da doença. 4,5 Embora comumente se manifeste em mulheres durante a terceira década de vida, a população pediátrica comumente é afetada. ${ }^{6}$

$\mathrm{Na}$ faixa etária pediátrica, a média da idade de início dos sintomas varia entre cinco e dez anos, tendo maior incidência na adolescência, com manifestação da doença entre 11-12 anos, com maior predomínio para o sexo feminino. ${ }^{7} \mathrm{~A}$ presença de história familiar de LES é encontrada em aproximadamente 11 a $35 \%$ dos casos. ${ }^{8,9}$

Sabe-se que a presença de linfócitos B hiperreativos e linfócitos T-supressor deficientes modificam a imunorregulação do organismo e promovem a formação de autoanticorpos, fazendo com que a manifestação da doença seja polimorfa, de evolução variável, com períodos de agravos e remissões. ${ }^{10,11}$

Estudos recentes evidenciam que o peróxido de hidrogênio desempenha um papel central na etiopatogênese do LES e pode predizer o risco para o desenvolvimento da doença. ${ }^{12,13}$

O peróxido de hidrogênio é capaz de iniciar o processo de apoptose celular através da ativação de fatores mitocondriais e induzir a condensação nuclear e posterior apoptose dos linfócitos. Ao se acumular no interior dos linfócitos B hiperreativos e linfócitos T-supressor são descritas " bombas metabólicas" que explodem em uma reação em cadeia e comprometem a glicogênese no ciclo de Krebs. Consequentemente, o processo para obtenção energética é prejudicado e essas células culminam para a morte programada. ${ }^{14}$
Estrógenos e prolactina são hormônios imunomoduladores que intervêm no processo da autoimunidade. ${ }^{15}$ Esses hormônios interferem na maturação das células $B$ e consequentemente na produção de anticorpos. Em relação às causas hormonais, não apenas o estrogênio e o androgênio estão ligados ao desenvolvimento da doença, como também fatores relacionados à dosagem sérica de prolactina e a deficiência de vitamina D são descritos como pilares importantes para o acionamento da autoimunidade. ${ }^{16,17}$

Estudos revelam que a vitamina D apresenta importante papel sobre a regulação e a diferenciação de células como linfócitos $\mathrm{T}$ e $B$ que participam de forma fundamental na formação de anticorpos. A vitamina $\mathrm{D}$ também afeta os linfócitos Th17 que secretam ocitocinas e IL-17 que participam da fisiopatogenia de doenças autoimunes. ${ }^{18}$

As características clínicas são polimórficas e de evolução crônica, com períodos de exacerbação e remissão da doença. ${ }^{19,20} \mathrm{~A}$ atividade da doença pode ser avaliada pela combinação de anamnese, exame físico e exames laboratoriais. ${ }^{21}$

Os principais anticorpos antinucleares relacionados à doença são anticorposanti-dsDNA, contra proteínas ribonucleares (anti-Smith-anti$\mathrm{Sm}$ e anti-RNP) e polimerases de RNA (antiRNA polimerases). ${ }^{17} \mathrm{~A}$ elevação dos títulos do FAN e dos níveis de anti-dsDNA foi incorporada aos critérios diagnósticos posteriormente.

Os critérios diagnósticos da doença foram recentemente revisados, com o objetivo de aumentar a acurácia diagnóstica (Quadro 1), em que a presença de quatro ou mais critérios clínicolaboratoriais definem a presença da doença. $\mathrm{O}$ índice SLICC (Systemic Lupus International Collaborating Clinics, 2012) é validado para o acompanhamento ambulatorial dos pacientes com LES para representar dano cumulativo pela doença. $^{22}$

Pericardite é a manifestação cardíaca mais comum, podendo ser clínica ou subclínica, e ocorre em até $55 \%$ dos pacientes acometidos. $\mathrm{O}$ derrame pericárdico geralmente é pequeno $\mathrm{e}$ 


\section{Quadro 1}

\section{Critérios SLICC para diagnóstico de LES}

1. Lupus cutâneo agudo: inclui rash malar, lupus bolhoso, e rash fotossensivel;

2. Lupus cutâneo crônico: rash disoide, hipertrófico ou pariculite lúpica;

3. Úlceras orais: palato, boca e língua; ou úlceras nasais;

4. Alopecia não cicatricial;

5. Sinovite de duas ou mais articulações, com edema ou derrame articular (ou artralgia, e rigidez matinal maior que 30 minutos);

6. Serosite: dor pleurítica típica por mais de um dia ou derrame pleural ou atrito pleural; dor pericárdica típica por mais de um dia ou efusão pericárdica ou atrito pericárdico ou eletrocardiograma com sinais de pericardite;

7. Renal: relação entre proteína e creatinina urinárias (ou proteinúria de 24 horas) com mais de $500 \mathrm{mg}$ de proteínas nas 24 horas, ou cilindros hemáticos;

8. Sistema Neurológico: convulsão, psicose, mielite; mononeurite múltipla, neuropatia cranial ou periférica, estado confusional agudo;

9. Anemia hemolítica;

10. Leucopenia $<4.000 / \mathrm{mm}^{3}$ ou linfopenia $<1.000 / \mathrm{mm}^{3}$, na ausência de outra causa conhecida;

11. Trombocitopenia $<100.000 / \mathrm{mm}^{3}$, na ausência de outra causa conhecida;

12. Fator antinuclear positivo;

13. Anticorpo anti-DNA positivo;

14. Anticorpo anti-Sm positivo;

15. Positividade de anticorpos antifosfolipídeos;

16. Complemento reduzido (frações $\mathrm{C} 3, \mathrm{C} 4, \mathrm{CH} 50$ );

17. Coombs direto positivo (na ausência de anemia hemolítica).

Fonte: adaptado de Petri e colegas. ${ }^{1}$

LES - lupus eritematoso sistêmico; SLICC - Systemic Lupus International Collaborating Clinics.

detectável apenas por ecocardiografia, raramente evoluindo para tamponamento cardíaco ou pericardite constritiva. OecoDopplercardiograma é o exame mais acessível para avaliar a função cardíaca e permite avaliar o grau de disfunção do ventrículo e estimar a pressão de artéria pulmonar. As alterações ecocardiográficas mais comuns incluem o derrame ou espessamento pericárdicos, aumento de câmaras esquerdas, alteração da fração de encurtamento ventricular esquerda e identificação de trombos intracavitários.

A endocardite de Libman-Sacks (eLS) é a endocardite não infecciosa que acomete cerca de $11 \%$ dospacientesportadoresdeLES.Caracterizase por vegetações estéreis nas válvulas cardíacas, especialmente a válvula mitral. Cursa comumente de forma assintomática, porém pode gerar embolia arterial sistêmica. Sabe-se que a utilização de corticoide e imunossupressores não apresenta efeitos satisfatórios sobre essas lesões valvares, entretanto, a anticoagulação deve ser utilizada para os pacientes que evoluíram negativamente com fenômenos tromboembólicos. O exame de escolha para a sua detecção é pelo ecoDopplercardiograma. $\mathrm{O}$ diagnóstico diferencial com endocardite infecciosa é imperativo pela elevada morbimortalidade e a antibioticoterapia deve ser instituída precocemente ao diagnóstico.

Envolvimento pulmonar ou pleural ocorre em cerca de $50 \%$ dos pacientes e a manifestação mais comum é pleurite com derrame de pequeno a moderado volume, geralmente bilateral.

O principal índice de atividade de doença atualmente utilizado para o LES é o SLEDAI (Systemic Lupus Erythematosus Disease Activity 
Index) que é composto por 24 itens incluindo sinais, sintomas e testes laboratoriais, cada qual recebendo um peso (variando de 1 a 8 ), de acordo com sua importância clínica ou gravidade. A soma final dos itens presentes correlaciona-se com a gravidade da doença, cujo grau máximo é de 105 pontos. Uma modificação desteíndice sem a inclusão dos testes laboratoriais é o SLEDAI$2 \mathrm{~K}$. Ambos os testes possuem boa aplicabilidade e vem sendo amplamente validado em diversos centros de referência. ${ }^{23}$

Os regimes terapêuticos disponibilizados para a população pediátrica, embora amplamente testados em adultos, podem não ser suficientes para controle da doença na população pediátrica e a escolha da opção utilizada depende dos órgãos e sistemas envolvidos, bem como a gravidade da doença. ${ }^{24} \mathrm{O}$ arsenal terapêutico disponibilizado inclui medicamentos de aplicações tópicas, antiinflamatório não esteroidal, corticoides (com ação anti-inflamatória e imunossupressora), medicamentos antirreumáticos modificadores da doença (DMARD), tais como hidroxicloroquina, metotrexate ou azatioprina, bem como imunossupressores. A hidroxicloroquina é o medicamento mais recomendado para o tratamento do LES juvenil, cuja dose em crianças não deve exceder $5 \mathrm{mg} / \mathrm{kg} / \mathrm{dia} .{ }^{17}$

O manejo adequado e a prevenção das infecções presentes têm papel crucial na sobrevida desses pacientes, sendo de suma importância a instituição de antibioticoterapia precocemente. Portanto, faz-se necessário prever e considerar riscos e prognósticos para que se possa eventualmente instaurar e promover medidas preventivas. A principal forma de intervir e prevenir agravos associados ao LES é o conhecimento e o monitoramento de fatores de risco, bem como das condições clínicas apresentadas durante as diversas fases da doença. ${ }^{14}$

Este trabalho visa aborda a temática do LES na infância e sua contextualização por meio de um relato de caso clínico através de uma revisão bibliográfica.

\section{Relato de Caso}

Criança do sexo feminino, 13 anos, sem comorbidades prévias e em acompanhamento regular com pediatra ambulatoriamente, foi admitida em emergência do Hospital Santa Izabel da cidade de Salvador-Bahia, referenciada por médico cardiologista por presença de derrame pericárdico moderado em um curso de 2 dias, evidenciado por meio de ecocardiograma de controle, em vigência de febre não mensurada por 8 dias. Emagrecida, não soube precisar a perda ponderal;apresentavadiscretataquidispneiaecom estadogeral regular, apresentando-se, naadmissão hospitalar, estável clínica e hemodinamicamente, respirando confortavelmente em ar ambiente, sem necessidade de oxigenoterapia complementar a despeito do desconforto respiratório. Aceitação parcial da dieta via oral, de padrão habitual, dejeções e diurese dentro do padrão de normalidade. Internamento recente (há 1 mês da admissão hospitalar) em outra unidade hospitalar para tratamento de provável pneumonia comunitária, fazendo uso de Ceftriaxone por 5 dias, e com término de antibioticoterapia com uso de Amoxacilina e Clavulanato em ambiente domiciliar, sem intercorrências. A pacientes informou que há cerca de 4 anos iniciou quadro de artralgia em mãos e eventual rash malar, sem prosseguir para investigações adicionais.

A tomografia de tórax à admissão apresentava pequeno derrame pleural livre à direita e pequeno a moderado derrame pleural, com sinais de loculação à esquerda. Consolidação pulmonar sugestiva de atelectasia passiva do lobo inferior esquerdo e segmento basal posterior do lobo inferior direito. Linfonodos proeminentes mediastinais e axilares, bilateralmente, o maior deles, pré vascular, medindo $1,7 \mathrm{~cm}$.

Iniciada antibioticoterapia com Cefepime e Vancomicina, entretanto cursou com rash difuso, sendo retirada a Vancominica e introduzido Teicoplamina na suspeita de provável reação alérgica. Foi realizado ciclo de Albendazol e Ivermectina. Os exames laboratoriais 
evidenciaram anemia $\mathrm{Hb} 7,4 \mathrm{~g} \backslash \mathrm{dL}$, coombs direto reagente, presença de anticardiolipina lúpica positiva e FAN 1:640 e demais achados dentro do padrão de normalidade.

Realizado novo ecocardiograma de controle no $5^{\circ}$ dia de internamento hospitalar, evidenciouse válvula mitral com imagem sugestiva de vegetações e refluxo de grau discreto a moderado. Derrame pericárdico difuso de grau importante, sem sinais de restrição diastólica. Achados extracardíacos com derrame pleural bilateral. Postergada a pulsoterapia para controle do LES por conta da presença de vegetações sugestivas de endocardite de Libman-Sacks. Cursou posteriormente com persistência da febre a despeito do uso de antibioticoterapia, sendo considerada a presença de endocardite bacteriana. Evoluiu com melhora progressiva das alterações cardíacas e pulmonares, em ganho ponderal e melhora da atividade de doença. Introduzida corticoterapia (predinisona com desmame ambulatorial), hidroxicloroquina, suplementação vitamínica com vitamina D com cálcio e AAS para prevenção de eventos tromboembólicos. Paciente recebeu alta hospitalar com continuidade da assistência médica em serviço de Home Care para término de antibioticoterapia por um curso de 6 semanas.

\section{Discussão}

Define-se LES como uma condição autoimune com processo fisiopatológico complexo, que envolve fatores genéticos, ambientais e possivelmente infecciosos envolvidos. ${ }^{16} \mathrm{~A}$ doença é caracterizada por interação complexa entre susceptibilidade genética e fatores ambientais, resultando primariamente em perda de tolerância imunológica e desenvolvimento de autoimunidade crônica. ${ }^{20,21}$

Os principais componentes do sistema imune inato e adaptativo, celular e humoral têm influência sobre a doença e pode acometer de forma variada diversos órgãos e tecidos. ${ }^{14}$

Por ser uma doença multissistêmica, muitas vezes de início insidioso e com apresentação clínica variável, o diagnóstico de lupus pode ser difícil, principalmente na avaliação inicial em serviço de emergência. ${ }^{5}$ Nenhuma alteração clínica ou laboratorial, isoladamente, faz o seu diagnóstico, apesar de algumas dessas alterações clínicas e laboratoriais apresentadas serem sugestivas de tal enfermidade. ${ }^{6}$

A percepção do estado de saúde e da qualidade de vida dos pacientes, bem como o impacto de sua doença e de seu tratamento, vem sendo amplamente reconhecidos como tópicos de pesquisa em estudos clínicos e epidemiológicos. Por essa razão, fazem-se necessárias a compreensão e utilização de escalas de qualidade de vida, também na prática clínica cotidiana. ${ }^{12}$

Em relação ao tratamento disponibilizado, este inclui o uso de glicocorticoide, antimaláricos e imunossupressores. ${ }^{1}$ Entretanto, alguns pacientes podem apresentar refratariedade à resposta terapêutica ou múltiplas manifestações sistêmicas que necessitam de tratamentos adicionais para controle da doença. Na população pediátrica, os efeitos colaterais são considerados maiores quando comparados à população adulta pelo maior tempo de duração da doença e exposição de efeitos cumulativos às drogas. ${ }^{14}$

Este caso clínico evidenciou paciente jovem com diagnóstico de LES, com complicações pulmonares e cardíacas, incluindo a endocardite de Libman-Sacks, ressaltando a importância por parte do pediatra sobre o conhecimento da doença e o impacto substancial na qualidade de vida de pacientes acometidos quando instituídas terapias precoces.

\section{Conclusão}

Torna-se de fundamental importância o reconhecimento precoce das principais manifestações clínicas que acometem o LES na assistência da população pediátrica. Por se tratar de uma doença crônica, a supressão da atividade da doença, a prevenção contra o surgimento de danos orgânicos, bem como o de seus efeitos 
colaterais secundários aos fármacos utilizados contribuem positivamente na qualidade de vida de pacientes acometidos por tal enfermidade.

\section{Referências}

1. Petri M, Orbai AM, Alarcón GS, Gordon C, Merrill JT, Fortin PR, et al. Derivation and validation of the Systemic Lupus International Collaborating Clinics classification criteria for systemic lupus erythematosus. Arthritis Rheum. 2012;64(8):2677-86.

2. Tsokos GC, Lo MS, Costa Reis P, et al. New insights into theimmunopathogenesis of systemic lupus erythematosus. Nat RevRheumatol 2016; 12: 716 730.

3. Rose T, Dörner. Drivers of the immunopathogenesis in systemic lupus erythematosus. Best Pract Res Clin Rheumatol. 2017;31:321-333.

4. Chen PY, Chang CH, Hsu CC, Liao YY, Chen KT. Systemic lupus erythematosus presenting with cardiac symptoms. Am J Emerg Med. 2014;32(9):1117-9.

5. P. Mistry and M. J. Kaplan, "Cell death in the pathogenesis ofsystemic lupus erythematosus and lupus nephritis," ClinicalImmunology, vol. 185, pp. 59-73, 2017.

6. M. R. Elliott and K. S. Ravichandran, "The dynamics ofapoptotic cell clearance," Developmental Cell, vol. 38, no. 2,pp. 147-160, 2016.

7. Frostegård J. Prediction and management of cardiovascular outcomes in systemic lupus erythematosus. Expert Rev Clin Immunol. 2015;11(2):247-53.

8. Trachana M, Koutsonikoli A, Farmaki E, et al. Safety and efficacyof rituximab in refractory pediatric systemic lupus erythematosusnephritis: a single-center experience of Northern Greece.Rheumatol Int 2013; 33: 809-813.

9. Midgley A, Watson L, Beresford MW. New insights into the pathogenesisand management of lupus in children. Arch Dis Child 2014; 99: 563-567.

10. Ciccone MM, Zito A, Dentamaro I, Vestito D, Scicchitano P, Iacoviello M, et al. [Vitamin D deficiency and cardiovascular diseases]. G Ital Cardiol (Rome). 2015;16(1):16-20.

11. Correa Freitas E, Evelyn Karnopp T, de Souza Silva JM, et al. Vitamin D supplementation ameliorates arthritis but does not all eviates renal injury in pristane-induced lupus model. Autoimmunity. 2019;52(2):69-77.
12. Morbach H, Girschick HJ. [B-cell targeted therapy for children and adolescents with rheumatic diseases]. Z Rheumatol 2013; 72: 347-353.

13. Klumb EM, Silva CA, Lanna CC, Sato EI, Borba EF, Brenol JC, et al. [Consensus of the Brazilian Society of Rheumatology for the diagnosis, management and treatment of lupus nephritis]. Rev Bras Reumatol. 2015;55(1):1-21.

14. Couture J, Silverman ED. Update on the pathogenesis and treatment of childhood-onset systemic lupus erythematosus. Curr Opin Rheumatol 2016; 28: 488-496.

15. Yan S, Yim LY, Lu L, et al. MicroRNA regulation in systemic lupus erythematosus pathogenesis. Immune Netw. 2014;14(3): 138-148.

16. Bernal CB, Zamora LD, Navarra SV.Biologic therapies in systemic lupus erythematosus. Int J Rheum Dis 2015; 18: 146-153.

17. Groot N, de Graeff N, Avcin T, et al. European evidencebased recommendations for diagnosis and treatment of childhood-onset systemic lupus erythematosus: the SHARE initiative. Ann Rheum Dis 2017; 76: 17881796.

18. Lino K, Trizzotti N, Carvalho FR, Cosendey RI, Souza CF, Klumb EM, Silva AA, Almeida JR (2018) Pp65 antigenemia and cytomegalovirus diagnosis in patients with lupus nephritis: report of a series. J Bras Nefrol $40: 44-52$.

19. Leone A, Sciascia S, Kamal A, et al. Biologicals for the treatment of systemic lupus erythematosus: current status and emerging therapies. Expert Rev Clin Immunol 2015; 11: 109-116.

20. Thorbinson C, Oni L, Smith E, et al. Pharmacological management of childhood-onset systemic lupus erythematosus. Paediatr Drugs2016; 18: 181-195.

21-Ambrose N, Morgan TA, Galloway J, et al. Differences in diseasephenotype and severity in SLE across age groups. Lupus 2016; 25:1542-1550.

22. Navarra SV, Guzman RM, Gallacher AE, et al. Efficacy and safetyof belimumab in patients with active systemic lupus erythematosus:a randomised, placebo-controlled, phase 3 trial. Lancet 2011; 377: 22-721-731.

23. Jorge AM, Lu N, Zhang Y, et al. Unchanging premature mortalitytrends in systemic lupus erythematosus: a general population-based study (1999-2014). Rheumatology (Oxford). 2018; 23-57(2):337-344.

24. Long H, Yin H, Wang L, et al. The critical role of epigeneticsin systemic lupus erythematosus and autoimmunity.J Autoimmun. 2016;74:118-138. 